\title{
Parametric design and optimization of high speed train nose
}

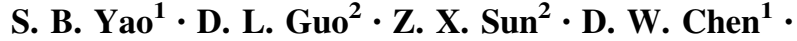 \\ G. W. Yang ${ }^{2}$
}

Received: 20 February 2014/Revised: 26 October 2014/ Accepted: 9 October 2015/

Published online: 18 November 2015

(C) Springer Science+Business Media New York 2015

\begin{abstract}
Aiming at shortening the design period and improve the design efficiency of the nose shape of high speed trains, a parametric shape optimization method is developed for the design of the nose shape has been proposed in the present paper based on the VMF parametric approach, NURBS curves and discrete control point method. 33 design variables have been utilized to control the nose shape, and totally different shapes could be obtained by varying the values of design variables. Based on the above parametric method, multi-objective particle swarm algorithm, CFD numerical simulation and supported vector machine regression model, multi-objective aerodynamic shape optimization has been performed. Results reveal that the parametric shape design method proposed here could precisely describe the three-dimensional nose shape of high speed trains and could be applied to the concept design and optimization of the nose shape. Besides, the SVM regression model based the multi-points criterion could accurately describe the nonlinear relationship between the design variables and objectives, and could be generally utilized in other fields. No matter the simplified model or the real model, the aerodynamic performance of the model after optimization has been greatly improved. Based on the SVR model, the nonlinear relation between the aerodynamic drag and the design variables is obtained, which could provide guidance for the engineering design and optimization.
\end{abstract}

Keywords Parametric design - Aerodynamic shape - SVM model · PSO - Multiobjective optimization $\cdot$ High speed trains

Z. X. Sun

sunzhenxu@imech.ac.cn

1 CSR Qingdao Sifang Locomotive Co., Ltd., Qingdao 266111, China

2 Key Laboratory for Application of Mechanics to Engineering Systems, Institute of Mechanics, Chinese Academy of Sciences, Beijing 100190, China 


\section{Introduction}

In recent years, the high speed trains worldwide have been rapidly developed and the running speed is getting higher and higher. Consequently, those aerodynamic effects which could be neglected in low speed conditions start to be significant, such as the aerodynamic drag, the pressure waves generated when two trains pass by each other in the tunnel or in the open air, the crosswind effects, and micro pressure waves. The bad effect could be greatly suppressed by the reasonable design of the nose shape. The high speed trains with their design speed higher than $300 \mathrm{~km} / \mathrm{h}$ include the Shinkansen series in Japan, ICE3 high speed train in German, the TGV series in France and CRH380 series in China, as shown in Fig. 1. Although different series correspond to totally different nose shapes, each series mainly focus on improving the aerodynamic performance in certain aspect and taking the national culture into consideration. Big slenderness ratio can be observed for the Shinkansen series, which could greatly decrease the tunnel pressure waves and micro pressure waves. While the slenderness ratios for ICE3 and TGV series are slightly smaller, which mainly take the aerodynamic lift and drag into consideration. CRH380A develops from the Shinkansen E2 series, takes the aerodynamic performance, national culture and manufacturing disciplines into consideration, and becomes one of the representatives for the new generation of high speed trains in China. Currently, the traditional design of the nose shape belongs to optimal selection, which needs detailed comparisons of a series of nose models by wind tunnel experiments, numerical simulations, or real vehicle tests. Consequently, a relatively long design period would be taken.

In order to improve the design efficiency of the nose shape and reduce the design cost, a lot of studies have been performed by researchers (Ku et al. 2010a; Jongsoo Lee 2008; Krajnovic 2009; Krajnovic et al. 2012; Cui et al. 2012; Yao et al. 2012a; $\mathrm{Ku}$ et al. 2010b; Vytla et al. 2010; Yao et al. 2012b). Restricted by the computer technologies and computational methods, early research on the nose shape usually focuses on single-objective optimization approaches for two-dimensional profiles (Ku et al. 2010; Jongsoo Lee 2008), which are hard applied to engineering application. In recent years, new progress has been made on three-dimensional nose shape optimization (Krajnovic 2009; Krajnovic et al. 2012; Cui et al. 2012; Yao et al. 2012; Ku et al. 2010; Vytla et al. 2010; Yao et al. 2012, 2014). Researches delivered by Krajnovic (Krajnovic 2009; Krajnovic et al. 2012), Ku et al. (2010), Vytla et al. (2010) and Yao (Yao et al. 2012, 2014) concentrate on multi-objective optimization of three-dimensional nose, which provide guidance on the real

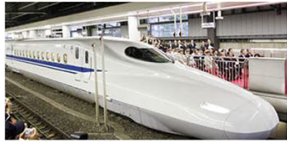

(a) E7

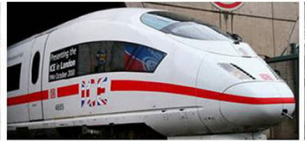

(b) ICE3

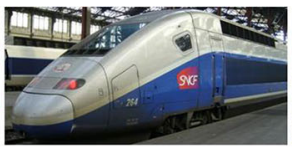

(c) TGV

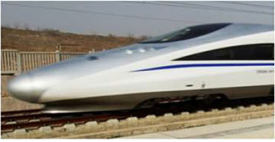

(d) CHR380A

Fig. 1 Kinds of nose of high speed trains 
application for the method with DOE and surrogate based modeling. However, most researches are limited in the already existing shape (Yao et al. 2012a, 2014) or very simplified shape (Krajnovic 2009; Krajnovic et al. 2012; Ku et al. 2010a; Vytla et al. 2010), and seldom relate to automatic design (Krajnovic et al. 2012) and optimization of the nose shape, which is the key problem when designing a new nose shape of high speed trains. In order to solve this problem, by combining the VMF parametric method, NURBS curves and discrete control point method, a parametric method to the nose shape has been proposed, which can deform the nose shape automatically. Based on this method, the multi-objective optimization of the nose shape has been performed by the combination of MOPSO, CFD and SVR.

\section{Parametric design approach}

As the development of computer technologies, computer aided design (CAD) plays a more and more important role in the design of high speed train nose, which may efficiently improve the design efficiency and design quality. Considering that the design of nose shape experiences several processes such as concept design, sketch design and detailed design, the introduction of parametric design into CAD could greatly reduce the cost caused by repeatedly shape revisions. Parametric shape design means that by revising the size of a part or a few parts, the corresponding shape could be revised automatically, so that the shape can be revised directly by the size of parts, in which the geometric information and topology information could be obtained automatically by the computer. This is one of the key technologies in shape optimization, and also is the key problem encountered when intelligently designing and optimizing the nose of high speed trains.

Although many parametric shape design software exist, and could design complex shapes parametrically, tedious operations are needed for these software and secondary developments are usually required for special problems. For aerodynamic shape optimization, Krajnovic et al. (2012) present a fully automatic multi-objective shape optimization method for improving the aerodynamic properties of trains, which shows a good idea for nose shape optimization. While the nose shape used in their studies is very simple and may not be used for engineering problems directly. Thus, a more practical parametrical method should be studied.

Due to the large slenderness ratio, the nose shape of high speed trains usually consist of complex free surfaces. When deformation is performed, the topologically
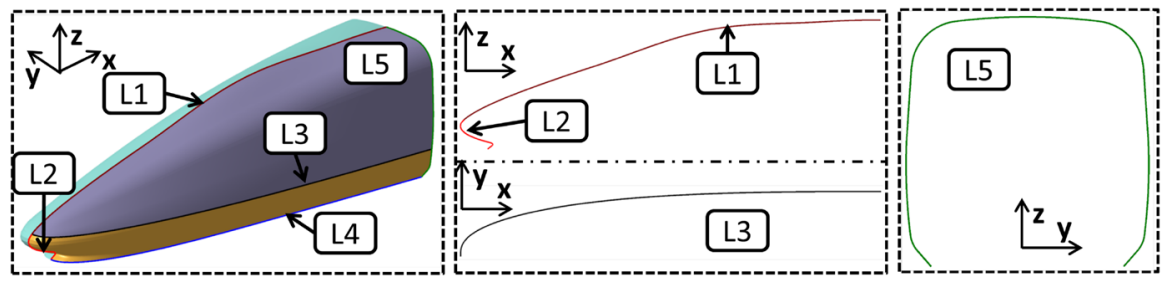

Fig. 2 Key control profiles of the nose shape 
different surfaces may be obtained, which will bring great difficulty to the shape deformation of high speed trains. Consequently, it is very necessary to develop parametric design method specifically for high speed trains. Figure 2 shows the key control profiles for the nose shape, in which the longitudinal profile is controlled by L1, the longitudinal profile of the cowcatcher is controlled by L2, the horizontal profile and bottom profile are controlled by L3 and L4, and the maximum crosssectional profile is controlled by L5. In general, the maximum cross-section is determined so as to match the existing train bodies. As a result, L5 will not be optimized and is given according to the maximum cross-section of CRH380A high speed trains. The profiles of L3 and L4 are similar and could be described with the same formula. L1 and L2 have the most influence on the nose shape. Totally different noses can be obtained via varying L1 and L2, especially the latter, which affects not only the aesthetic effect, but also the aerodynamic performance (Yao et al. 2012a, b). In the following sections the parametric method for each profile and surface will be described in detail.

\subsection{Vehicle modeling function}

The VMF parametric method is derived by J.H. Rho etc. (Rho et al. 2009) through further improvement on Bernstein polynomial. This method can greatly reduce the design variables and better describe the profiles with little curvature in the shape design of automobiles. Compared to NURBS method, this method can efficiently improve the design and optimization efficiencies of automobiles. Ku et al. (2010) introduced VMF method into the nose shape design of high speed trains and extracted four design variables to control the shape deformation. Although it is difficult to take the maximum cross-section and the volume of the nose into consideration through these variables and can hardly obtain any nose shape that could be applied into engineering application, it sheds light on the way to optimize the nose shape of high speed trains. In order to sufficiently utilize the advantage of the VMF method, proper improvement on this method has been performed and then be applied to the parametric design of L1, L3 and L4.

The basic form of two-dimensional profiles given by J.H. Rho is taken as:

$$
F\left(\frac{x}{c}\right)=\left(\frac{x}{c}\right)^{A_{1}}\left(1-\frac{x}{c}\right)^{A_{2}} S\left(\frac{x}{c}\right)+\left(1-\frac{x}{c}\right) Y_{1}+\left(\frac{x}{c}\right) Y_{2} .
$$

In which $x$ ranges from 0 to $c . Y_{1}$ and $Y_{2}$ are the vertical coordinates of the starting point and ending point, respectively. Different kinds of profiles can be obtained by changing the form of $S\left(\frac{x}{c}\right) . A_{1}$ and $A_{2}$ are the curvatures of the front and rear part of the profile. When $A_{1}$ ranges from 0 to 1 and $A_{2}$ is greater than 1 , the curvature at $\mathrm{x}=0$ for $F\left(\frac{x}{c}\right)$ tends to infinity, and at $\mathrm{x}=\mathrm{c}$ for $F\left(\frac{x}{c}\right)$ equals to 0 ; When $A_{2}$ ranges from 0 to 1 , the curvature at $\mathrm{x}=\mathrm{c}$ for $F\left(\frac{x}{c}\right)$ tends to infinity. Consequently, in order to avoid the infinite curvature at end points, cubic polynomial is adopted at the endpoints. The coefficients of the polynomial are determined by the coordinates and curvatures at the endpoints. When the curvature 
of the endpoints is finite values, in order to control the curvatures, proper revision is performed for formula (1) and takes the form as:

$$
F\left(\frac{x}{c}\right)=\left(\frac{x}{c}\right)^{A_{1}}\left(1-\frac{x}{c}\right)^{A_{2}} S\left(\frac{x}{c}\right)+G\left(\frac{x}{c}\right) .
$$

$G\left(\frac{x}{c}\right)$ is used to control the change of curvatures at endpoints and typically takes the form of a polynomial, of which the coefficients are determined by the curvatures and coordinates at the end points.

Figure 3 shows the exact curves for different forms of $G\left(\frac{x}{c}\right)$ when $S\left(\frac{x}{c}\right)=Y_{2}-Y_{1}$. It can be seen that after the revision of formula (1), the curvatures of the start point can be effectively controlled by varying the value of $A_{1}$. When $A_{2}$ is greater than 1 , the curvature of the endpoints keeps zero and don't vary with $A_{2}$.

The parametric formulas of L1, L3 and L4 take the form of formula (2), and are described in detail as follows:

The profile of L1 takes the form as:

$$
z(x)=\left(\frac{x-x_{11}}{x_{12}-x_{11}}\right)^{A_{11}}\left(1-\frac{x-x_{11}}{x_{12}-x_{11}}\right)^{A_{12}} a_{k 1}\left(1-\frac{x-x_{11}}{x_{12}-x_{11}}\right)^{a_{b 1}}+g(x) .
$$

In which $g(x)=2\left(z_{12}-z_{11}\right) \frac{x-x_{11}}{x_{12}-x_{11}}-\left(z_{12}-z_{11}\right)\left(\frac{x-x_{11}}{x_{12}-x_{11}}\right)^{2}$. It is used to control the heights and curvatures at endpoints. $x_{11}$ and $x_{12}$ are the X-coordinates of the start point and end point. $z_{11}$ and $z_{12}$ are the $\mathrm{z}$-coordinates of the start point and end point.

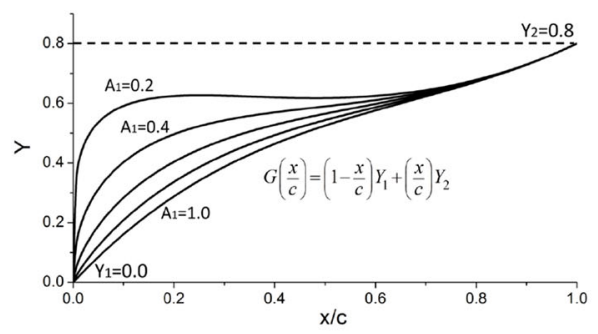

(a)

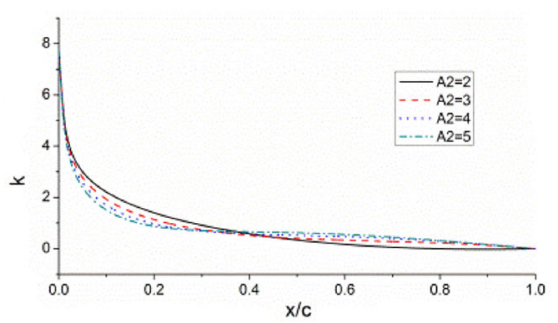

(c)

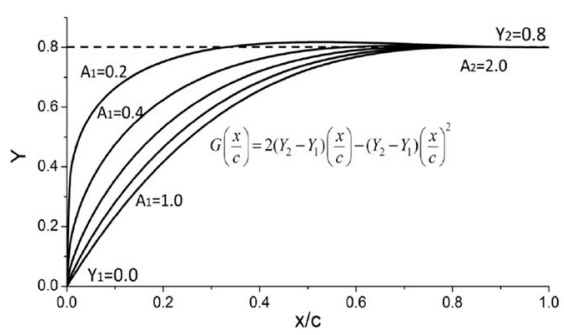

(b)

Fig. 3 Curves of different forms and curvatures. a $G\left(\frac{x}{c}\right)=\left(1-\frac{x}{c}\right) Y_{1}+\left(\frac{x}{c}\right) Y_{2} ; \mathbf{b} G\left(\frac{x}{c}\right)=2\left(Y_{2}-Y_{1}\right)\left(\frac{x}{c}\right)-$ $\left(Y_{2}-Y_{1}\right)\left(\frac{x}{c}\right)^{2}$; c Different curvatures for different values of $\mathrm{A}_{2}$ 
The profile of L3 takes the form as:

$$
y(x)=\left(\frac{x-x_{21}}{x_{22}-x_{21}}\right)^{A_{21}}\left(1-\frac{x-x_{21}}{x_{22}-x_{21}}\right)^{A_{22}} a_{k 2}\left(\frac{x-x_{21}}{x_{22}-x_{21}}-1\right)^{a_{b 2}}+g(x) .
$$

In which $g(x)=2\left(y_{22}-y_{21}\right) \frac{x-x_{21}}{x_{22}-x_{21}}-\left(y_{22}-y_{21}\right)\left(\frac{x-x_{21}}{x_{22}-x_{21}}\right)^{2}$. It is used to control the heights and curvatures at endpoints. $x_{21}$ and $x_{22}$ are the $\mathrm{x}$-coordinates of the start point and end point. $y_{21}$ and $y_{22}$ are the y-coordinates of the start point and end point;

The profile of L4 takes the form as:

$$
y(x)=\left(\frac{x-x_{31}}{x_{32}-x_{31}}\right)^{A_{31}}\left(1-\frac{x-x_{31}}{x_{32}-x_{31}}\right)^{A_{32}} a_{k 3}\left(\frac{x-x_{31}}{x_{32}-x_{31}}-1\right)^{a_{b 3}}+g(x) .
$$

In which $g(x)=2\left(y_{32}-y_{31}\right) \frac{x-x_{31}}{x_{32}-x_{31}}-\left(y_{32}-y_{31}\right)\left(\frac{x-x_{31}}{x_{32}-x_{31}}\right)^{2}$. It is used to control the heights and curvatures at endpoints. $x_{31}$ and $x_{32}$ are the $\mathrm{x}$-coordinates of the start point and end point. $y_{31}$ and $y_{32}$ are the $y$-coordinates of the start point and end point.

\subsection{NURBS}

Although only a few design variables could be utilized to obtain different kinds of profiles for VMF method, the profiles are limited for VMF and it seems very difficult to parametrically design complex profiles. For the parametric design of the profile of the cowcatcher, a new kind of profiles with sufficient variations is needed. The use of non-uniform rational B-spline curves is one of the frequently used methods in parametric shape design. Any arbitrary profile could be obtained by reasonably setting the number, coordinates and weights of control points. Consequently, this approach is adopted for the parametric design of L2.

Three-order NURBS curve is adopted for L2 and consists of 5 control points, just as Fig. 4 shows. In order to control the length of the streamline, a quadratic polynomial is added for the $\mathrm{x}$-coordinate in the NURBS curve, which takes the form as:
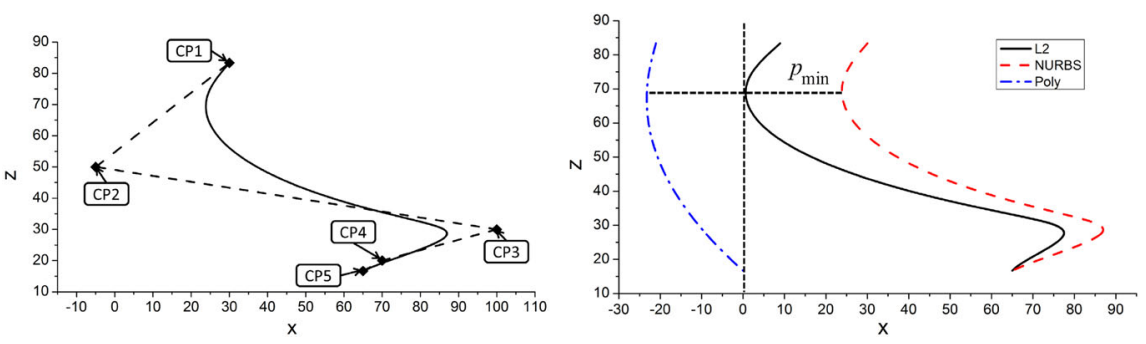

Fig. 4 Modified NURBS curves 


$$
x(z)=p_{x}(u)+g(z)
$$

where $g(z)=A(z-H)^{2}+B$. The minimum value of $p_{x}(u)$ is no less than $0 . H$ is the value of $\mathrm{z}$ when $p_{x}(\mathrm{u})=p_{\min }$. The coefficient $A$ controls the width of the polynomial and is determined by the $\mathrm{x}$-coordinate of CP5. $B$ is controlled by the $\mathrm{x}$-coordinate of CP1. In order to reduce the number of design variables, the weight in the formula is constant for simplicity.

\subsection{Approximation for free surfaces}

\subsubsection{Surface fitting}

The nose shape of high speed trains is controlled by key two-dimensional profiles. A simple method is adopted in the present paper to fit the surface. Considering the two-dimensional profiles as the borders, the surface of the nose could be divided into four regions. Since the nose is symmetric for the $y=0$ plane, only half of the nose needs to be considered for surface deformation. The surface deformation is driven by the two-dimensional profiles. Each surface could be considered as a spatial quadrilateral composed by four borders. The coordinates of the surface could be obtained by linear fitting with the formula (7) and (8):

$$
\begin{gathered}
y(x)=y_{\text {min }}(x)+\frac{x}{l(x)} y_{\text {max }}(x) \\
z(x)=z_{\text {min }}(x)+\frac{x}{l(x)} z_{\text {max }}(x)
\end{gathered}
$$

The coordinates obtained by (7) and (8) may be different with the coordinates at the borders. As a result, further revision should be performed so as to obtain the final smooth surface.

\subsubsection{The shape of the cab window}

The design of the cab window determines the view of the drivers. The inclination angle, the space, and the transition between the cab window and the streamline
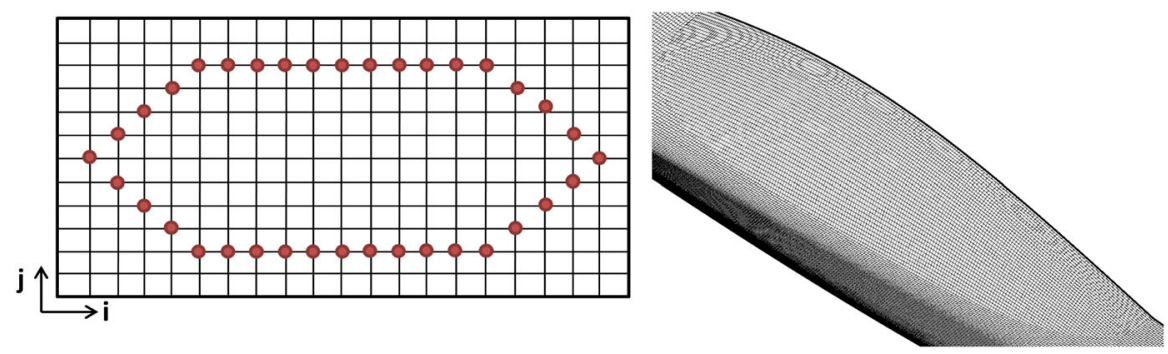

Fig. 5 Schematic of parametric design of the cab window 
should be all considered during the design. Since all the surfaces are approximated by discrete points, the borders of the cab window are also obtained by discrete points, just as shown in Fig. 5.

The coordinates of the borders are determined by formula (9), where $g c$ controls the shape of the borders. The shape of the cab window is determined by formula (10), where $g h$ controls the height of the window, $g n_{1}$ the smooth transition between the window and side of the nose, $g n_{2}$ the smooth transition between the window and upper and lower parts of the nose.

$$
\begin{gathered}
y_{i d}=\left(y_{\text {hmax }}-y_{\text {hmin }}\right)\left[1-\left(\frac{x-x_{\text {hmin }}}{x_{\text {hmax }}-x_{\text {hmin }}}\right)^{g c}\right]^{1 / g c} \\
z(x, y)=g h\left(\sin \left(\pi\left(y_{i}-y\right) /(2 y)\right)\right)^{g n_{1}}\left(\sin \left(\pi\left(x-x_{\min }\right) /\left(x_{\max }-x_{\min }\right)\right)\right)^{g n_{2}}
\end{gathered}
$$

In the above formula, $x_{\min }$ and $x_{h \min }$ are the maximum value and minimum value of the cab window in $x$ direction respectively. Similarly, $y_{h \min }$ and $y_{h \min }$ are the maximum value and minimum value in $y$ direction respectively. Since the cab window is symmetric in $\mathrm{y}=0$ plane, $y_{h \min }$ equals 0 .

\subsubsection{The design of the drainage}

The shape design of the drainage on the nose not only affects the aesthetic effect, but also affects the fluid characteristics around the nose. Consequently, parametric design of the drainage is also introduced in the present paper. The shape of the drainage could be obtained by superposing the increments of y-coordinate on the original surfaces, which could be calculated by formula (11) and (12).

$$
\begin{gathered}
h_{\text {nose }}(x)=h_{n m} \sin \left(\frac{x-x_{\text {nmin }}}{x_{\text {nmax }}-x_{\text {nmin }}} \pi\right) \\
\operatorname{delty}_{\text {nose }}(x, z)=h_{\text {nose }}(x)\left(\sin \left(\frac{z-z_{n \min }}{z_{\text {nmax }}-z_{\text {nmin }}} \pi\right)\right)^{n n s}
\end{gathered}
$$

The variation of $\mathrm{x}$ coordinate of the drainage could be described by the formula (11), while the variation of $\mathrm{z}$ coordinate could be described by the formula (12). $h_{n m}$ controls the maximum variation of the drainage, and nns controls the transition between the drainage and the basic surface. $x_{\text {nmin }}, x_{\text {nmax }}, z_{\text {nmin }}$ and $x_{\text {nmax }}$ control the position of the drainage.

\subsubsection{Laplace smoothing}

All obtained surfaces belong to quadrilateral surfaces, which may cause unsmooth connections at adjacent boundaries. Consequently, it is very necessary to introduce the mesh smoothing technique. The Laplace smoothing method is based on umbrella principle, and can smooth the surface by defining an umbrella operator on the smoothing vertex. Standard Laplace smoothing method is a widely used 
technique, which determines the direction of adjustment by defining a Laplace operator on each vertex, and moves the vertex with a certain speed in this direction. The formula of this approach takes the form as:

$$
P_{\text {new }}=P_{\text {old }}+\lambda L\left(P_{\text {old }}\right)
$$

where $P_{\text {new }}$ is the vertex after smoothing, $P_{\text {old }}$ the vertex before smoothing, $\lambda$ the speed adjustment factor, taking a value between 0 and 1 .

The Formula of Laplace operator $L\left(P_{\text {old }}\right)$ takes the form as:

$$
L(P)=\frac{1}{\sum_{j \in D(p)} w_{j}} \sum_{j \in D(p)} w_{j}\left(Q_{j}-P\right)
$$

where $w_{j}$ is the weight of vertex $Q_{j}$ which is a first-order neighborhood of vertex $P$, and usually takes the value of $w_{j}=\left\|Q_{j}-P\right\|^{-1}$.

Figure 6 shows the flow chart of parametric designing the nose shape of high speed trains. Firstly the formulas of two-dimensional profiles are determined. Then the basic original shape could be approximated by key control profiles. By the use of the Laplace smoothing method, a smooth surface could be obtained. Thirdly the basic shape of the cab window and the drainage could be superposed on the original shape, resulting in the final nose shape.

\subsection{The extraction of design parameters}

The extraction of key parameters can finally determine the aerodynamic performance of parametric nose. In order to obtain the nose with excellent aerodynamic performance and vision effects, proper number of parameters is extracted according to the above two criteria. Some parameters may have little effect on the aerodynamic performance of high speed trains, but may have an important influence on the geometry of the nose, so the sensitivity analysis of parameters is not provided in this paper. The design variables that control the nose shape are listed in Table 1 . There are 33 variables in total, 20 variables of which control the key two-dimensional profiles while the rest 13 variables control the shape of the auxiliary surfaces.

For different nose shapes, the connection part to the train body usually keeps the same. As a result, the variables A12, A22 and A33, which control the wake shape, are kept constant. Only 30 variables are needed to be optimized for the parametric design.

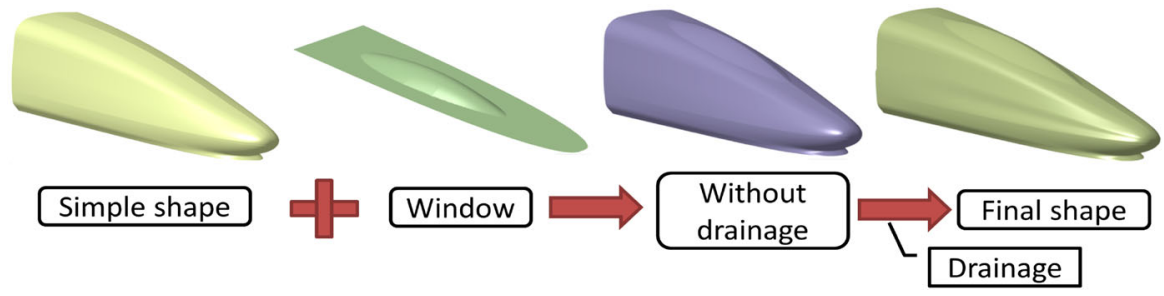

Fig. 6 Flow chart of parametric designing the nose shape of high speed trains 
Table 1 Design variables that control the nose shape

\begin{tabular}{lllll}
\hline Deformation & & & & \\
\hline Longitudinal profile of the nose & $A_{11}$ & $A_{12}$ & $a_{k 1}$ & $a_{b 1}$ \\
Horizontal profile of the nose & $A_{21}$ & $A_{22}$ & $a_{k 2}$ & $a_{b 2}$ \\
Profile of the bottom & $A_{31}$ & $A_{32}$ & $a_{k 3}$ & $a_{b 3}$ \\
Profile of the cowcatcher & & & & \\
CP1 & $x_{p 1}$ & - & - & - \\
CP2 & $x_{p 2}$ & - & $z_{p 2}$ & - \\
CP3 & $x_{p 3}$ & - & $z_{p 3}$ & - \\
CP4 & $x_{p 4}$ & - & $z_{p 4}$ & - \\
CP5 & $x_{p 5}$ & - & - & - \\
Shape of the cab window & $g h$ & $g c$ & $g n_{1}$ & $g n_{2}$ \\
Position of the cab window & $x_{h m i n}$ & $x_{h \max }$ & $y_{h m a x}$ & - \\
The drainage & $h_{n m}$ & $n n s$ & - & - \\
Position of the drainage & $x_{n \text { min }}$ & $x_{n \text { max }}$ & $z_{n \text { min }}$ & $z_{n \text { max }}$ \\
\hline
\end{tabular}

Taking these 30 variables as the design variables, the multi-objective optimization is performed to optimize the aerodynamic drag and the volume of the nose.

\subsection{Nose shape approximation test}

Figure 7 shows different noses of high speed trains. It can be seen that totally different streamlines can be obtained by adjusting the design variables. The basic outline of the nose could be sketched by three profiles which controls the nose shape. The profile of the cowcatcher zone greatly enriches the nose shape that could be further adjusted by the shape of the cab window and the drainage. In general, the parametric design approach proposed in the present paper could describe the threedimensional nose shape in detail, and could be used for the concept design and optimization design of the nose.

\section{Numerical method}

\subsection{CFD}

The influence on aerodynamic drag and the volume of the nose by the change of the shape is mainly concerned in the present paper. A simplified model is considered by

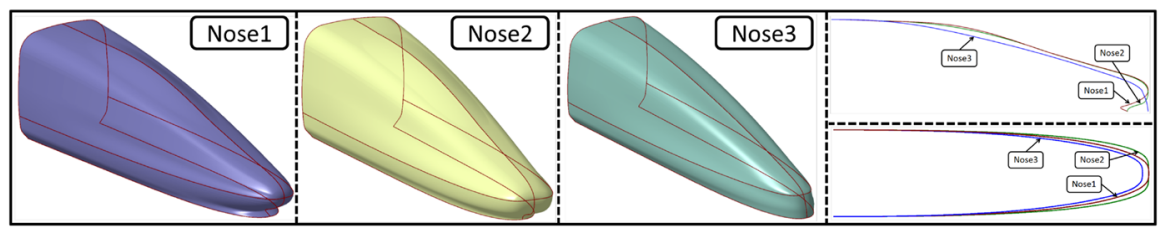

Fig. 7 Different streamlines of high speed trains 
portioning the geometry in three groups, and only the trailing bogies are considered, while other auxiliary components are neglected, just as shown in Fig. 8. The aerodynamic drag is obtained by CFD analysis, while the volume of the nose is obtained by self-developing codes. In this paper, the speed of high-speed train is $300 \mathrm{~km} / \mathrm{h}$, so the Mach number is 0.245 . Under this condition, the air compression characteristic has an obvious effect on the aerodynamic drag of the train. Therefore, the steady compressible Reynolds-averaged Navier-Stokes equations based on the finite volume method are used to predict the aerodynamic drag. Roe's FDS scheme is used to calculate convective fluxes, and Lower-Upper Symmetric GaussSeidel (LU-SGS) is chosen for temporal discretization. The k- $\omega$ SST model is selected as the turbulence model. The standard wall functions are used near the wall so that the accuracy of the CFD results could be ensured with a limited amount of mesh.

Computational domains and boundary conditions: taking the length of the simplified train as the characteristic length $\mathrm{L}$, then the length of inflow direction is $1 \mathrm{~L}$, the length of outflow direction is $2 \mathrm{~L}$, the width is $1 \mathrm{~L}$, the distance between the bottom of the train and the ground is $0.00235 \mathrm{~L}$ and the height is $0.5 \mathrm{~L}$, as shown in Fig. 9. The flow velocity is $83.33 \mathrm{~m} / \mathrm{s}$; the far-field pressure is $1 \mathrm{~atm}$, the temperature is $288 \mathrm{~K}$ and the reference area is the maximum cross-sectional area of the train. As a result of the compressibility calculation model, the one-dimensional inviscid flow of the Riemann invariants is introduced as the far-field boundary conditions, which are also known as non-reflective boundary conditions. Inflow, outflow and the top boundaries are all set as far-field boundary conditions and the train body is a non-slip wall. The ground is treated as the moving wall so as to simulate the ground effect, and the moving speed is equal to the train speed.

\subsection{Mesh independence validation}

Hexahedral grids are adopted for mesh generation. Prism grids are distributed along the wall, of which $y+$ of the first layer should range from 30 to 100 to ensure the use of wall functions. Since mesh distribution greatly affects the numerical results, four suits of mesh distributions are comparatively analyzed to obtain the reasonable mesh distribution, as shown in Fig. 10.

The results are shown in Table 2. It can be seen that the drag coefficients obtained by the four kinds of mesh distributions get no big difference. However, as the mesh size grows bigger, the lift coefficient of the trailing car gets relatively smaller. Consequently, the mesh distribution with an amount of 12.12 million grids is adopted for analysis, which could not only ensure the accuracy of numerical results, but also is endurable for calculations of multiple cases.

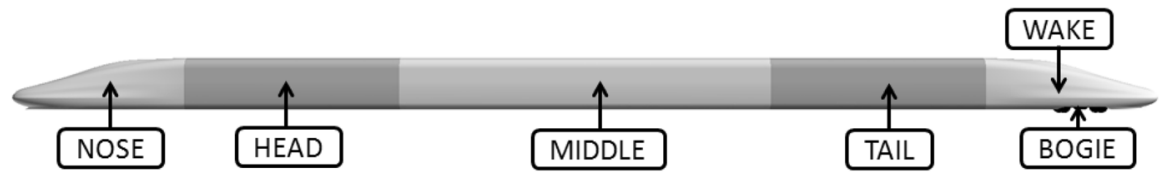

Fig. 8 Geometry of the simplified train model 

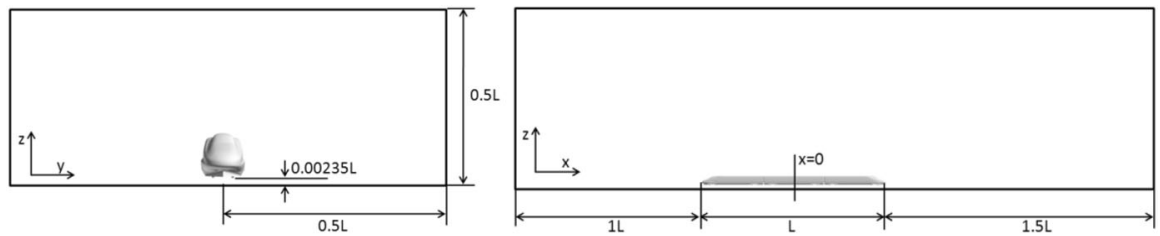

Fig. 9 Computational domain
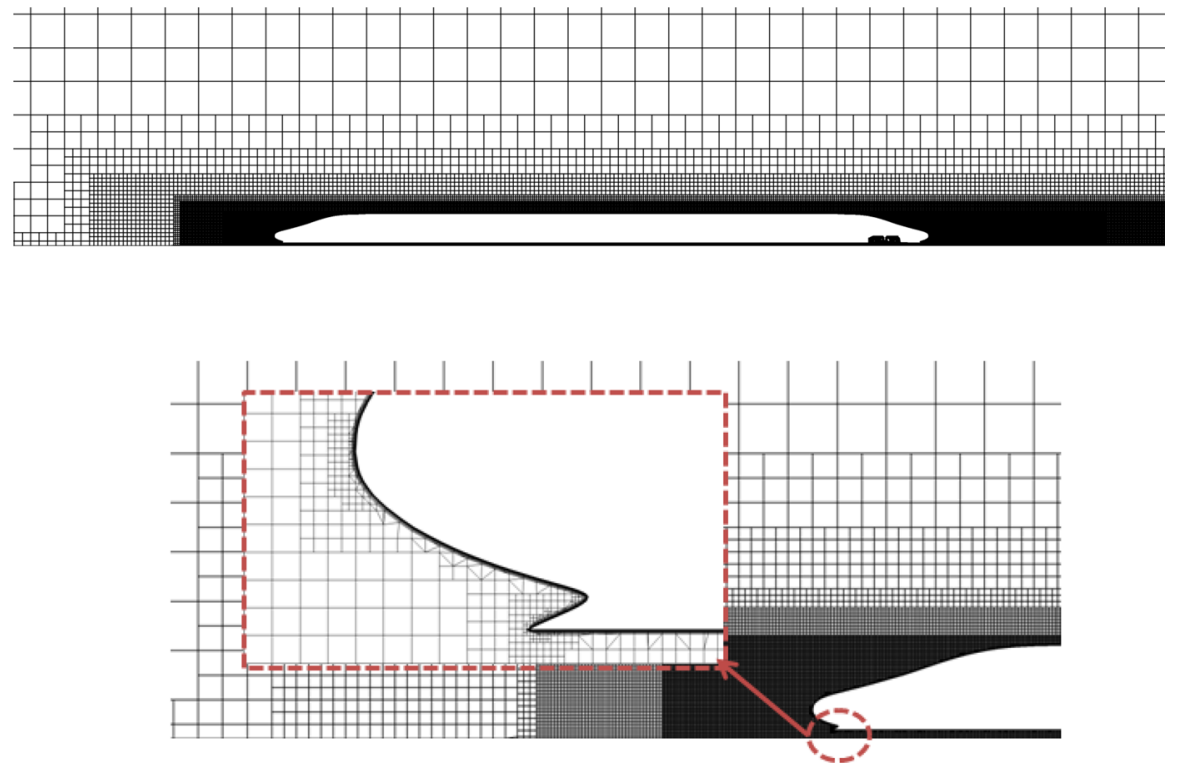

Fig. 10 Meshes for CFD

\section{Multi-objective aerodynamic optimization of the nose shape}

\subsection{Selection of sample points}

The running environment of high speed trains is very complex, and the nose shape has a serious effect on a series of design objectives, such as the aerodynamic drag, the volume of the driver's cab, the aerodynamic lift of the trailing car, the micropressure wave generated when the train passes a tunnel and the aerodynamic noise. It is unbearable to take all of these objectives into consideration because of the expensive CFD cost. For a new shape of high speed trains, the aerodynamic drag and the volume of the nose are two basic design objectives to be considered firstly, since the less the aerodynamic drag becomes, the less energy is consumed for the train operation; the bigger the volume of the nose is, the more comfort the driver obtains. Thus, only the aerodynamic drag and the nose volume are taken to be optimal objectives in this paper, and the methodological allegations will be discussed one by one in next sections. 
Table 2 Mesh distributions and corresponding CFD results

\begin{tabular}{lllllll}
\hline $\begin{array}{l}\text { Mesh number/ } \\
\text { million }\end{array}$ & $\begin{array}{l}\text { Thickness/ } \\
\mathrm{mm}\end{array}$ & $\begin{array}{l}\text { Layer } \\
\text { number }\end{array}$ & $\begin{array}{l}\text { Minimum size/ } \\
\mathrm{mm}\end{array}$ & $\begin{array}{l}\text { Stretching } \\
\text { ratio }\end{array}$ & $\begin{array}{l}\text { Total } \\
\mathrm{Cd}\end{array}$ & $\begin{array}{l}\text { Tail- } \\
\mathrm{Cl}\end{array}$ \\
\hline 9.03 & 0.8 & 10 & 12 & 1.2 & 0.201 & 0.059 \\
12.12 & 0.8 & 10 & 6 & 1.2 & 0.202 & 0.064 \\
16.26 & 0.8 & 10 & 3 & 1.2 & 0.204 & 0.065 \\
30.52 & 0.8 & 10 & 3 & 1.2 & 0.205 & 0.066 \\
\hline
\end{tabular}

A quasi CRH380A model (Nose 1 in Fig. 7) is chosen as the prototype to facilitate the comparative analysis. The design variables range $50 \%$ of the original values. Since the shape variation around the cowcatcher is relatively larger, the eight corresponding design variables range $200 \%$ of the original values. In order to obtain a more reasonable distribution of training samples in the design space, the Latin hypercube sampling method based on Max-minimum criterion is adopted in the present paper. 70 sampling points is obtained, 66 of which are chosen as initial training points and the rest four are set as testing points. Since the multi-points method is utilized to build the final response surface, the number of final training points is greater than 66 , which will be described in the following section.

\section{2 \&-TSVR}

The support vector machines (SVM) model is based on the structural risk minimization principle, and could be widely used in high dimensional problems and non-linear problems. In order to control the generalization capability, SVM needs to control the values of experience risks and confidence range during the learning process. Taking the training error as the constraints and the minimal value of confidence range as the objective, SVM finally turns into solving a convex quadratic programming problem with linear constraints. Non-linear mapping is firstly used by the SVM model to map the data into a higher-dimensional space, then linear regression is performed in the higher-dimensional space. Consequently, non-linear regression in the original space could be ensured. Sometimes the optimal hyperplane is difficult to calculate directly in the characteristic space during the mapping process, since the dimension of the space is growing dramatically. In order to solve this problem, the kernel function is introduced in the SVM model, so that the calculation could be performed in the input space.

Several regression methods (Shao et al. 2013; Peng 2010) could be found for SVM model. The $\varepsilon$-TSVR ( $\varepsilon$-twin support vector regression, $\varepsilon$-TSVR) algorithm proposed by Shao et al. (2013) is utilized in the present paper. Compared to the standard SVR algorithm (Vapnik 1998) and TSVR algorithm (Peng 2010), ع-TSVR gets more accurate prediction ability and needs less training time. For the non-linear regression problem, the basic problems for $\varepsilon$-TSVR algorithm can be found in (Shao 2013). 
The dual problems of $\varepsilon$-TSVR algorithm are (15) and (16). The details could be referred to in the literature (Shao et al. 2013).

$$
\begin{array}{ll}
\max _{\boldsymbol{\alpha}} & -\frac{1}{2} \boldsymbol{\alpha}^{T} \boldsymbol{H}\left(\boldsymbol{H}^{T} \boldsymbol{H}+c_{3} \boldsymbol{I}\right)^{-1} \boldsymbol{H}^{T} \boldsymbol{\alpha}^{T}-\left(\boldsymbol{e}^{T} \varepsilon_{1}+\boldsymbol{Y}^{T}\right) \boldsymbol{\alpha}+\boldsymbol{Y}^{T} \boldsymbol{H}\left(\boldsymbol{H}^{T} \boldsymbol{H}+c_{3} \boldsymbol{I}\right)^{-1} \boldsymbol{H}^{T} \boldsymbol{\alpha} \\
\text { s.t. } & \mathbf{0} \leq \boldsymbol{\alpha} \leq c_{1} \boldsymbol{e} . \\
\max _{\gamma} & -\frac{1}{2} \boldsymbol{\gamma}^{T} \boldsymbol{H}\left(\boldsymbol{H}^{T} \boldsymbol{H}+c_{4} \boldsymbol{I}\right)^{-1} \boldsymbol{H}^{T} \gamma^{T}-\left(\boldsymbol{Y}^{T}-\boldsymbol{e}^{T} \varepsilon_{2}\right) \boldsymbol{\gamma}-\boldsymbol{Y}^{T} \boldsymbol{H}\left(\boldsymbol{H}^{T} \boldsymbol{H}+c_{4} \boldsymbol{I}\right)^{-1} \boldsymbol{H}^{T} \boldsymbol{\gamma} \\
\text { s.t. } & \mathbf{0} \leq \boldsymbol{\gamma} \leq c_{2} \boldsymbol{e} .
\end{array}
$$

where

$$
\begin{gathered}
\boldsymbol{H}=\left[\boldsymbol{K}\left(\boldsymbol{A}, \boldsymbol{A}^{T}\right) \boldsymbol{e}\right] \\
\boldsymbol{v}_{1}=\left(\boldsymbol{H}^{T} \boldsymbol{H}+c_{3} \boldsymbol{I}\right)^{-1} \boldsymbol{H}^{T}(\boldsymbol{Y}-\boldsymbol{\alpha}) \\
\boldsymbol{v}_{2}=\left(\boldsymbol{H}^{T} \boldsymbol{H}+c_{4} \boldsymbol{I}\right)^{-1} \boldsymbol{H}^{T}(\boldsymbol{Y}+\boldsymbol{\gamma})
\end{gathered}
$$

and $\boldsymbol{v}_{1}=\left[\begin{array}{ll}\boldsymbol{u}_{1}^{T} & b_{1}\end{array}\right]^{T}, \boldsymbol{v}_{2}=\left[\begin{array}{ll}\boldsymbol{u}_{2}^{T} & b_{2}\end{array}\right]^{T}$. By solving (15) and (16), $\boldsymbol{u}_{1}, \boldsymbol{u}_{2}, \boldsymbol{b}_{1}$ and $\boldsymbol{b}_{2}$ could be obtained, the predicting values of the SVM model could be obtained by (20).

$$
f(\boldsymbol{x})=\frac{1}{2}\left(f_{1}(\boldsymbol{x})+f_{2}(\boldsymbol{x})\right)=\frac{1}{2}\left(\boldsymbol{u}_{1}^{T}+\boldsymbol{u}_{2}^{T}\right) \boldsymbol{K}(\boldsymbol{A}, \boldsymbol{x})+\frac{1}{2}\left(b_{1}+b_{2}\right) .
$$

Given the specific training samples, the free coefficients of the SVR model are $c_{1}$, $c_{2}, c_{3}, c_{4}, \varepsilon_{1}, \varepsilon_{2}$ and $\sigma$, which are crucial to the generalization capability of the SVR model. However, no theoretical basis could be found to rigorously calculate these variables till now. The cross validation method and PSO method are combined to determine these variables in the present paper. To simplify the problem, we assume $c_{1}=c_{2}$ and $c_{3}=c_{4}$. As a result, only 5 free coefficients are needed to determine. For a given set of free coefficients, the convex quadratic programming problems (15) and (16) are needed to be resolved twice. In order to improve the training efficiency, the over relaxation iteration technique is introduced by Shao $\mathrm{YH}$ in literature (Shao et al. 2013), which is also adopted in the present paper.

Figure 11 shows the optimization process for the free coefficients of SVR model, which are described in detail as follows:

(1) For the given training sample set, random division of 1 groups is firstly performed depending on the exact circumstance. Make sure that the number of training samples in each group is same.

(2) Initial coefficients (such as the population and the number of iterations) should be given. These coefficients greatly affect the optimization efficiency, and should not be too big or too small. The population is 35 and the number of iterations is 200 in the present paper. 
(3) Each group is chosen sequentially as the test samples while the rest groups are utilized to construct the SVR model. Then the prediction error $\% R M S E_{\mathrm{i}}$ could be obtained. The fitness function of PSO algorithm could be obtained by (21):

$$
f i t=\frac{1}{l} \sum_{i=1}^{l} \% R M S E_{i}
$$

where 1 is the number of groups and $\% R M S E_{i}$ is the prediction error when the i-th group acts as the test samples, which takes the form as:

$$
\% \text { RMSE }=100 \sqrt{\frac{1}{n_{s}} \sum_{i=1}^{n_{s}}\left(y_{i}-y_{i}^{(p)}\right)^{2}} / \frac{1}{n_{s}} \sum_{i=1}^{n_{s}} y_{i}
$$

In which $y_{\mathrm{i}}$ is the real value, $y_{i}^{(p)}$ the prediction value by SVR and $n_{s}$ the number of test samples.

(4) The optimal values of the free coefficients can be obtained after iterations. When using the SVR model to predict the objective values, the average value of the values predicted by each sub-SVR model is considered as the final prediction result. Since two objectives exist in the present paper, two SVR models are needed.

\subsection{Multi-objective PSO algorithm}

As we know, there are many optimization approaches for us to solve optimization problems, but it is difficult for us to select the best one to solve the nose shape optimization problems. The SVR is adopted in this paper, so the main propose of adopting the optimization approach is to find the non-dominated solutions but the efficiency of the approach is not highly required. Multi-objective PSO is one of the popular optimization approaches to get the non-dominated solutions of complex optimization problems, and combined with Genetic Algorithm, it has been used for shape optimization of high speed train nose (Vytla et al. 2010).

The particle swarm optimization (PSO) algorithm is proposed by Kennedy, etc. (Kennedy et al. 1995) in 1995. This algorithm is easy to achieve and get an excellent

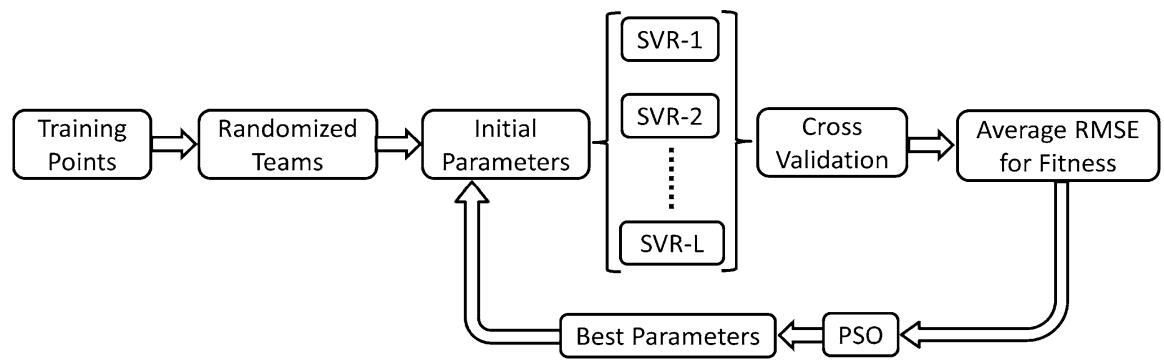

Fig. 11 Flow chart of the construction of SVR model 
capability in global optimization. Moore (Moore and Chapman 1999) firstly used this approach to solve multi-objective problem in 1999. Then kinds of multiobjective PSO algorithms are developed, such as Parsopoulos et al. (2002), Hu et al. (2002), Konstantinos et al. (2004). Every algorithm has its own features, and should be chose carefully for different optimal problems. Based on the non-inferiority classification, Li (2003) introduced the niche technique and crowding distance into the PSO method, and constructed a new algorithm NSPSO, which gets stronger optimization capability than NSGA-II. The exterior file is introduced into NSPSO in the present paper. The niche particle number and crowding distance are also adopted to judge the quality of the particles in the exterior file. The particle with the least niche particle number and the largest crowding distance in the exterior file is chosen as the optimal particle of the population. Consequently, a modified particle swarm optimization method (MPSO) is constructed in this paper. Figure 12 shows the construction process of MPSO. In the present paper, the population of particles is 200 , the number of iterations is 2000 , the accelerating factor is 2 , the inertia factor generally changes from 1.2 to 0.4 with the iteration and the maximum speed of the particles is 0.05 .

\section{Results and discussions}

\subsection{The construction of SVR model}

The analysis of each sample by CFD is extremely cost. Till now the reasonable number of initial training samples is hard to determine by theoretical derivation. The number of design variables is 30 and the number of initial training samples is 66 . However, it cannot be ensured the SVR model constructed from the initial training samples meet the accuracy requirement. If the number of initial training samples is large enough to ensure the accuracy requirement, the calculation cost from CFD analysis would become considerably large and would reduce the optimization efficiency. With regards to this, a multi-point approach is adopted in this paper. If the accuracy of the SVR is not satisfied, 6 samples from the non-dominated set are chosen for CFD analysis. After obtaining the values of the objectives of these 6 samples, these samples are added to the initial training set and the SVR model is reconstructed, until the prediction accuracy of the model is achieved. Figure 13 shows the distribution of non-dominated sets during the iteration. It can be seen that

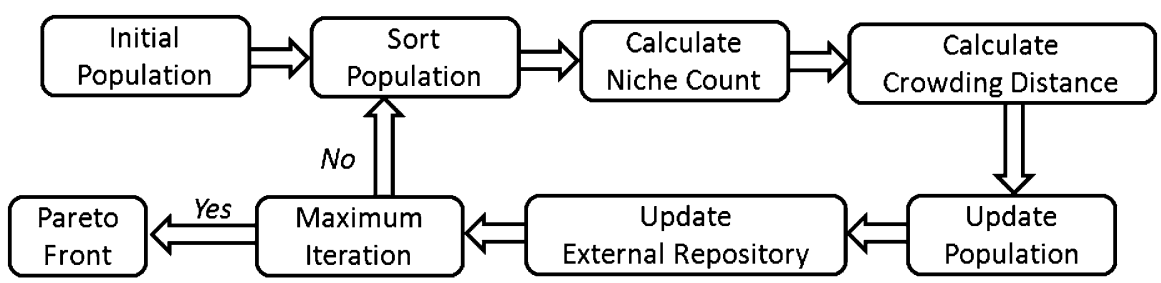

Fig. 12 Flow chart of the MPSO algorithm 
the volume values of non-dominated set- 1 obtained from the initial training set are bigger than the non-dominated sets obtained by adding points, indicating that the added points have an important influence on the predicting error of SVR model. Five point additions have been performed. As a result, 30 additional points have been added to the initial training sample set and the final SVR model is constructed by 96 points. When the cross validation approach is performed, every group is composed of two points. As the number of training points becomes larger, the number of groups also becomes larger. Consequently, the training time becomes longer. However, compared to the cost of CFD analysis, the time cost of training the SVR model could be neglected.

The prediction accuracy of the SVR model is determined by the following three factors: the fitness value when the iteration of PSO stops, which can describe the prediction accuracy of the whole design space; the prediction error of the test samples, which can represent the generalization capability of the SVR model; the prediction error of the non-dominated set, which can describe the prediction accuracy around the optimal points. When the values of above three are all below $5 \%$, the SVR model can be seen as an accurate model. Figure 14a shows the average fitness and prediction error of test points for the two objectives during the iteration. It can be seen that the average fitness and the prediction error are both less than $5 \%$, and the prediction accuracy of the volume is obviously higher than that on the drag. Within the whole design space, the fluctuation of the drag is considerably stronger than that of the nose volume, and the nonlinear relationship between the design variables and the aerodynamic drag is more complex than that between the design variables and the nose volume. It can be seen from Fig. 14b that during the beginning of the iteration the prediction error around the non-dominated set is slightly larger. However, after adding points for the third time, the prediction error reaches a value within $5 \%$, while the prediction error of the drag is still a little large. After adding points for the fifth time, the prediction accuracy of SVR model reaches the design requirement.

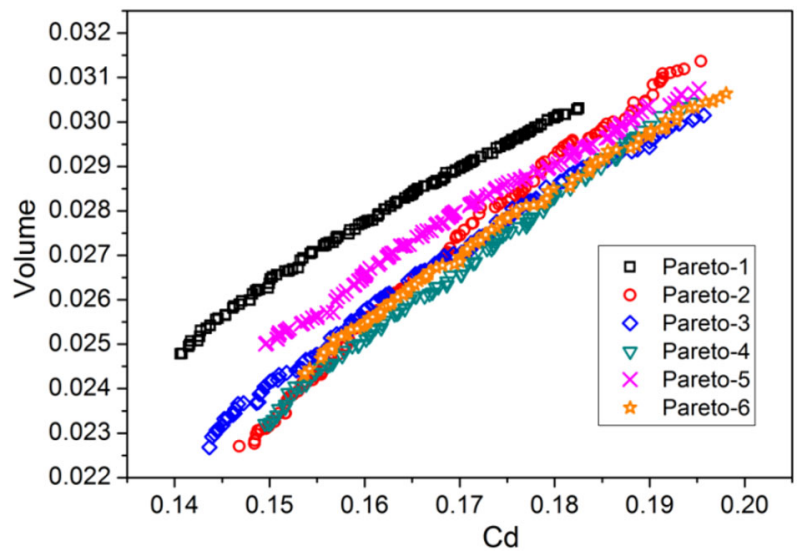

Fig. 13 Non-dominated sets during each iteration 


\subsection{Aerodynamic analysis}

Figure 15 shows the final non-dominated set, which contains 126 design points. If all the points are validated by CFD analysis, the computational cost could be unbearable. As a result, six design points are chosen to perform the comparative analysis. It can be seen that these six points are evenly distributed in the nondominated set. The aerodynamic drag and volume are both increasing with the design point number.

It can be seen from Fig. 16 that the topologies of the six points are the same. The nose is the region with largest variation. When the volume of the nose is relatively small, a sharp nose could be detected and the width of the nose is a little smaller, just as Case 1 shows; if the volume of the nose is a little larger, the curvature of the profile in front of the nose is very small, resulting in larger bluntness. The width of the nose gets increased, even exceeds the maximum width of the train body. The original cowcatcher is curved forward. However, it is curved backward for all the cases after optimization. It can be deduced that the backward cowcatcher will aid in reducing the aerodynamic drag.

Table 3 shows the aerodynamic drag and the volume of the original shape and 6 typical design points. Compared to the original design, the volume of Case 1 reduces by $1.85 \%$, while the volumes of the others all increases; the aerodynamic drags of the whole train for optimal cases are all reduced, in which Case 1 reduces the most, and reaches $19.31 \%$; Case 6 reduces the least, and only reduces by $1.49 \%$. The lifts of the trailing car for all optimal cases are reduced greatly, in which the lift in Case 1 becomes negative, the lifts of Case 2 and Case 3 are close to zero, and the lift in Case 6 reduces the least, but still reaches a value of $37.5 \%$. The aerodynamic drag of the leading car for all optimal cases is reduced. The drag of the trailing car in Case 1 and Case 5 reduces a little, while the drag in Case 6 increases by $4.17 \%$. Case 2 is chosen as the final optimal shape in the present paper, so that the volume performance and the aerodynamic performance for each car are all improved.

Figure 17 shows the pressure contour around the nose of the optimal shape and original shape. It can be observed that an apparent high pressure zone exists around
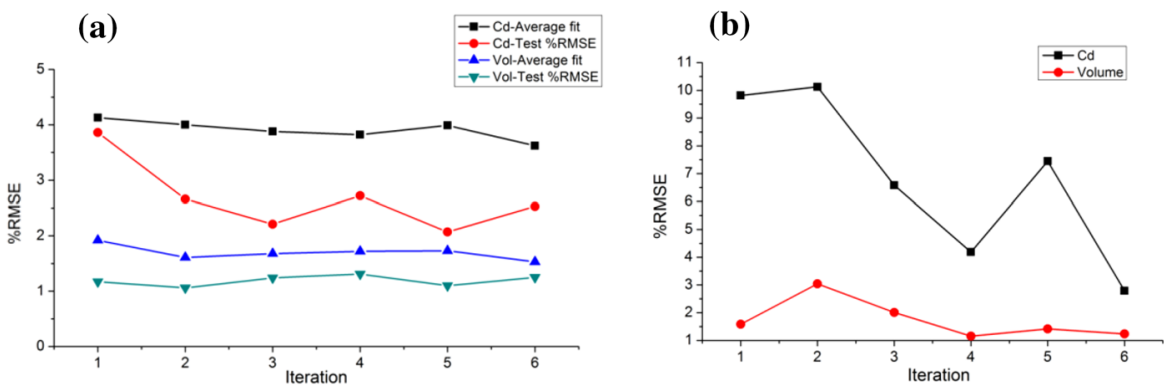

Fig. 14 a Average fitness and prediction error of the test samples for each iteration; b prediction error of the non-dominated set [six design points are chosen, and the prediction error is calculated by formula (22)] 


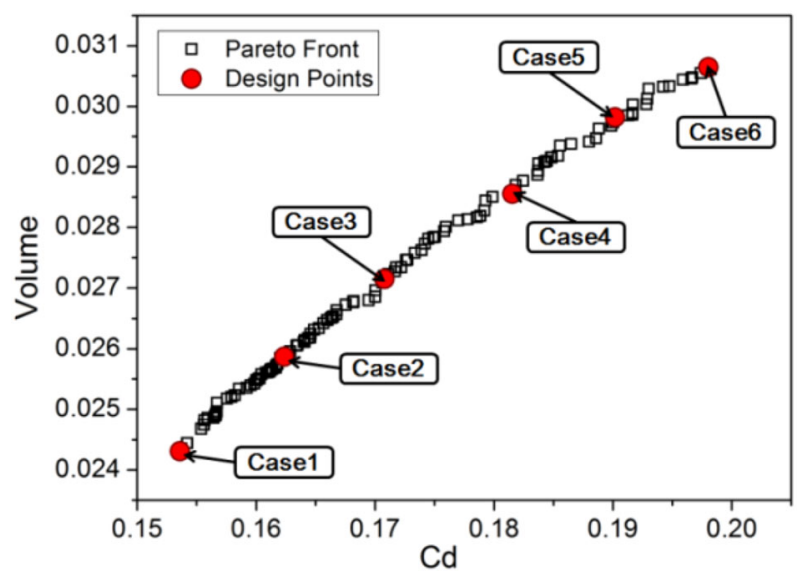

Fig. 15 Non-dominated set and typical samples

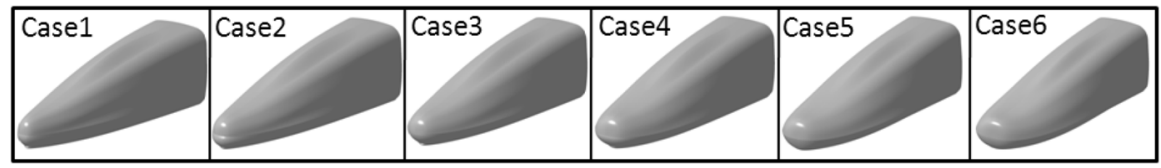

Fig. 16 Nose shape for each case

Table 3 The aerodynamic drag and the volume of the original shape and 6 typical design points

\begin{tabular}{lccccc}
\hline & Volume $\left(\mathrm{m}^{\wedge} 3\right)$ & Total-Cd & Tail-Cl & Head-Cd & Tail-Cd \\
\hline Original & 87.64 & 0.202 & 0.064 & 0.062 & 0.096 \\
Case 1 & 86.02 & 0.163 & -0.010 & 0.051 & 0.068 \\
Reduction & $1.85 \%$ & $19.31 \%$ & $115.63 \%$ & $17.74 \%$ & $29.17 \%$ \\
Case 2 & 90.79 & 0.170 & 0.004 & 0.052 & 0.074 \\
Reduction & $-3.59 \%$ & $15.84 \%$ & $93.75 \%$ & $16.13 \%$ & $22.92 \%$ \\
Case 3 & 94.45 & 0.173 & 0.006 & 0.053 & 0.076 \\
Reduction & $-7.77 \%$ & $14.36 \%$ & $90.63 \%$ & $14.52 \%$ & $20.83 \%$ \\
Case 4 & 98.32 & 0.182 & 0.023 & 0.054 & 0.085 \\
Reduction & $-12.17 \%$ & $9.90 \%$ & $64.06 \%$ & $12.90 \%$ & $11.46 \%$ \\
Case 5 & 103.82 & 0.192 & 0.035 & 0.055 & 0.093 \\
Reduction & $-18.46 \%$ & $4.95 \%$ & $45.31 \%$ & $11.29 \%$ & $3.13 \%$ \\
Case 6 & 107.14 & 0.199 & 0.040 & 0.056 & 0.100 \\
Reduction & $-22.25 \%$ & $1.49 \%$ & $37.50 \%$ & $9.68 \%$ & $-4.17 \%$ \\
\hline
\end{tabular}

the nose. As the bluntness of the nose is increased, the high pressure zone grows bigger. However, the curvature of zone 1 becomes greater, which makes the intensity of negative pressure much stronger. The cowcatcher becomes backward after optimization, and the slope becomes smaller, resulting in a smaller high 
pressure zone. Consequently, the nose after optimization attributes to reduce the intensity of the high pressure, and further reduce the aerodynamic drag of the leading car.

Figure 18 shows the pressure coefficient $(C p)$ on the longitudinal profile of the leading nose for the original shape and optimal shape. Since the bluntness of the nose after optimization grows obviously larger, apparently negative zone exists in zone 1 . Then the coefficient keeps the same for the original shape and optimal shape. The pressure coefficient on the wake for the original shape and the optimal shape keeps almost the same, except that the positive pressure after optimization is a little larger, which could be neutralized with the negative pressure on the surface of the nose, so that the inviscid drag of the trailing car could be reduced.

Figure 19 shows the distribution of vertex cores in the wake zone for the original shape and optimal shape. It can be seen that the size of vortex cores gets obviously smaller for the optimal shape, especially V3 and V6. V6 disappears after optimization, while the intensity of V3 gets apparently weaker. The high pressure zone enlarges, so that the inviscid drag of the train could be greatly reduced. The big vertex structures V1 and V2 are also reduced after optimization. Meanwhile, the distance between V1 and V2 gets shortened, which could weaken the influence on the surroundings.

\subsection{Comparison of the real train model}

The above optimization of the nose shape is based on a simplified train, so that the cost of mesh generation and CFD analysis can be greatly reduced. A proper simplification had been performed. Only the bogies beneath the nose had been reserved while other ancillary components are all neglected. However, these components impose an intense effect on the aerodynamic performance of the whole train. As a result, comparative study on the real train model has been performed to determine whether the optimal shape could be used in engineering application. The authors have already performed a study on the windshield and found that it has a strong influence on the distribution of aerodynamic drag for each carriage while gets

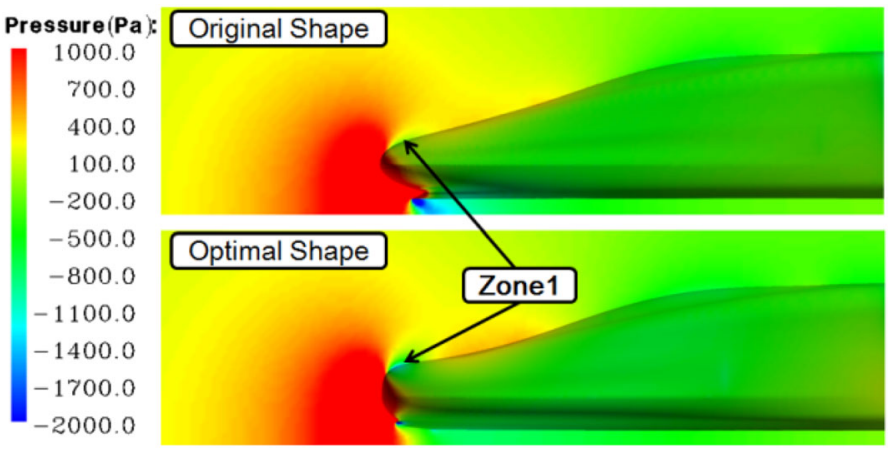

Fig. 17 The pressure contour around the nose of the optimal shape and original shape 

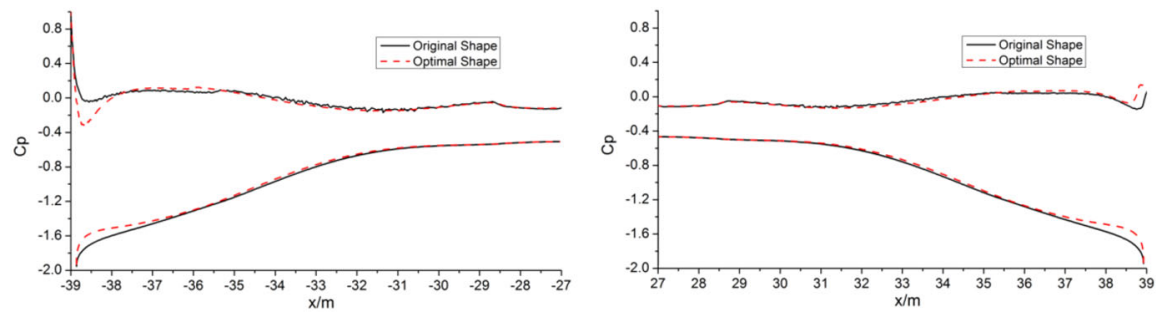

Fig. 18 The pressure coefficient $(\mathrm{Cp})$ on the longitudinal profile of the nose for the original shape and optimal shape

no big influence on the drag of the whole train. Since the drag of the whole train is mainly concerned in the present paper, the fully enclosed outside windshields are adopted. The shape of the train is shown in Fig. 20.

The distribution of aerodynamic forces of the original train and the optimal train has been shown in Table 4. It could be obviously seen that the drag of the real model is greatly larger than the simplified one and the aerodynamic lift of the optimal real train also gets increased. Compared to the original train, the total drag of the optimal train has been reduced by $13.62 \%$, in which the drag of the leading car has been reduced by $25.41 \%$, the drag of the middle car has been increased by $1.47 \%$, and the drag of the trailing car has been reduced by $8.99 \%$. The lift of the trailing car has been reduced by $9.84 \%$. The reduction of aerodynamic drag for each car gets big difference with that of the simplified train. The biggest reduction of drag could be observed on the leading train, while the reduction of drag for the whole train is almost the same.

\subsection{Nonlinear relationship between the aerodynamic forces and the design variables}

The parametric approach proposed in the present paper is very suitable for the module design of the nose shape. The influence on the objectives by each design variable could shed light on the engineering design of the components of the nose. After constructing the SVR model, it is very convenient to obtain the relationship
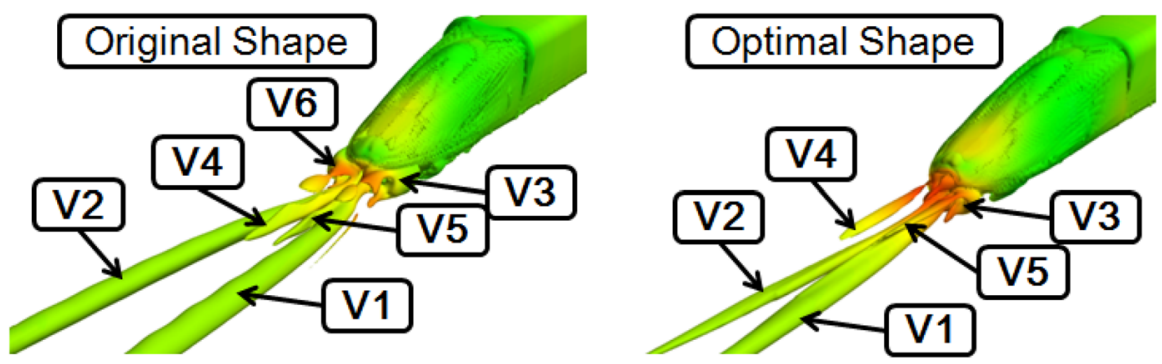

Fig. 19 Distribution of vertex cores in the wake zone for the original shape and optimal shape 


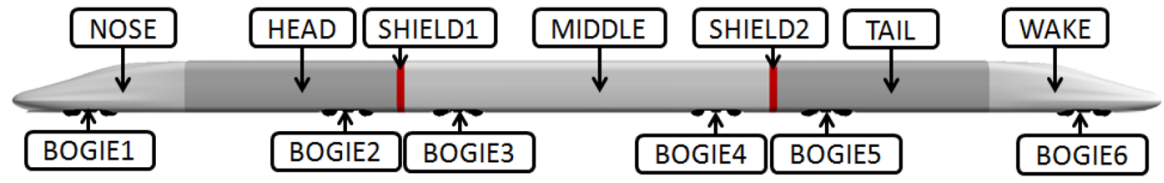

Fig. 20 Geometry of the real train model

Table 4 The distribution of aerodynamic forces of the original train and the optimal train

\begin{tabular}{lclccc}
\hline & Total-Cd & Tail-Cl & Head-Cd & Middle-Cd & Tail-Cd \\
\hline Original & 0.279 & 0.061 & 0.122 & 0.068 & 0.089 \\
Case 2 & 0.241 & 0.055 & 0.091 & 0.069 & 0.081 \\
Reduction & $13.62 \%$ & $9.84 \%$ & $25.41 \%$ & $-1.47 \%$ & $8.99 \%$ \\
\hline
\end{tabular}

between the aerodynamic drag and design variables. Figure 21 shows the relationship between the drag and three design variables that control the main profiles. The $\mathrm{x}$-coordinate is normalized for comparison. When the design variable concerned varies, the other variables keep the same with the original shape. Same handling of Figs. 22 and 23 is performed. It can be seen that the relationships between the drag and the three design variables are similar and certain nonlinear characteristics could be observed. $A_{11}$ and $a_{k 1}$ control the curvature at the tip of the nose together. Taking $A_{11}$ as an example, as $A_{11}$ increases, the curvature tends to be small, the nose tends to be blunt, and the aerodynamic drag begins to decrease. As $A_{11}$ reaches a certain value, the drag tends to increase. $a_{b 1}$ controls the curvature of the profile that connects the nose and the cab window. As $a_{b 1}$ increases, the concavity there tends to be stronger, the negative zone begins to enlarge, which could reduce the inviscid drag of the leading car. If $a_{b 1}$ is large enough, flow separation may arise and increase the inviscid drag of the leading car. $A_{21}$ and $A_{31}$ control the curvatures of horizontal profile and bottom profile respectively. As both variables increase, the curvature of the corresponding profile decreases at the tip of the nose, resulting in smaller bluntness of the nose. As a result, the aerodynamic drag is monotonically decreasing with $A_{21}$ and $A_{31}$. It can be seen that the aerodynamic drag of the train is positively correlated with the bluntness of the nose in a fairly large extent. The drag of the train is quasi linear with $a_{k 2}$, which mainly influences the curvature of the horizontal profile at the tip and middle of the nose. As $a_{k 2}$ increases, these zones expand outward, resulting in larger width and blunter of the nose. Consequently, the frontal area of the nose enlarges, so that the drag of the nose increases. $a_{b 2}$ controls the width of the nose. As $a_{b 2}$ increases, the width of the nose decreases, so that the drag of the train decreases. $a_{k 3}$ mainly affects the curvature of the bottom profile at the tip and middle of the nose. As $a_{k 3}$ increases, these zones expand outward, resulting in larger volume at the bottom, which could retard the flow around the cowcatcher and result in a bigger drag of the nose. Different from $a_{k 3}, a_{b 3}$ mainly affects the curvature of the bottom profile in the middle of the nose. As $a_{b 3}$ decreases, the width of the bottom increases. Since the shape of the front and back of the nose keeps unchanged, the flow around the 

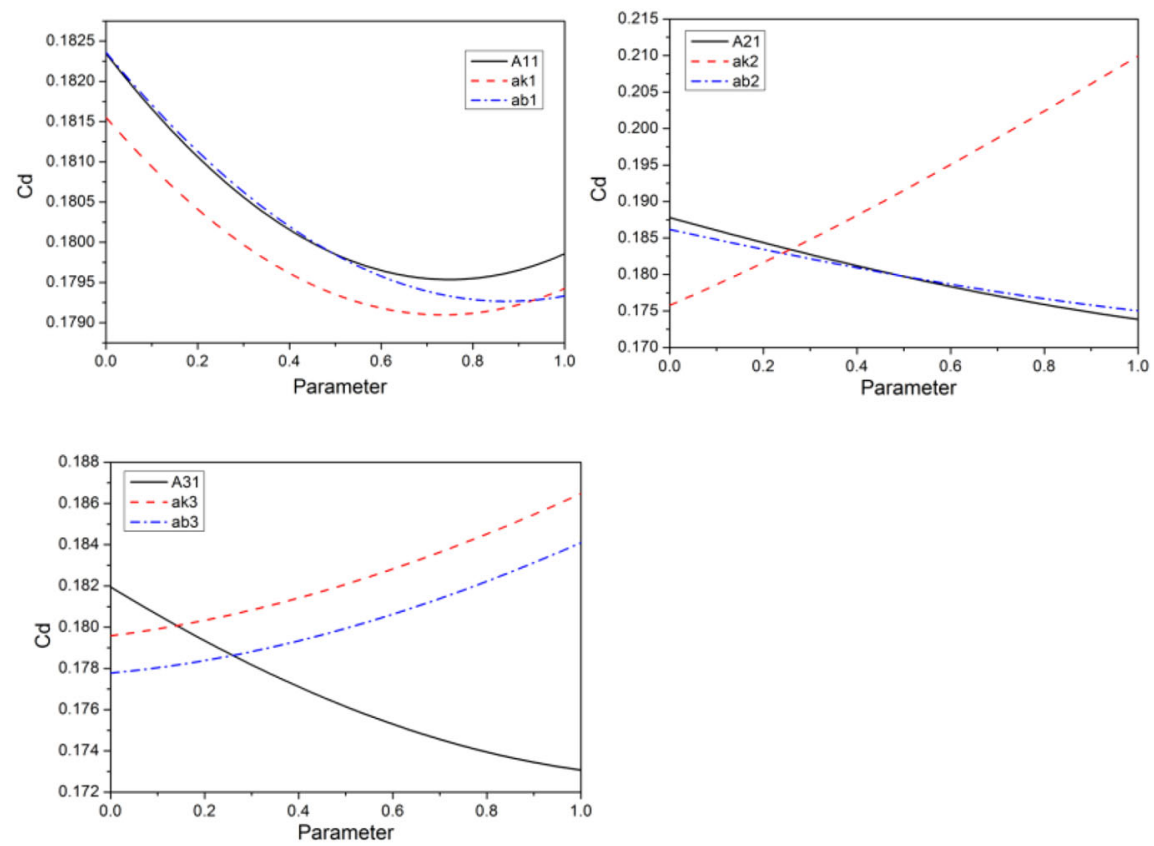

Fig. 21 Relationship between the drag and three design variables that control the main profiles
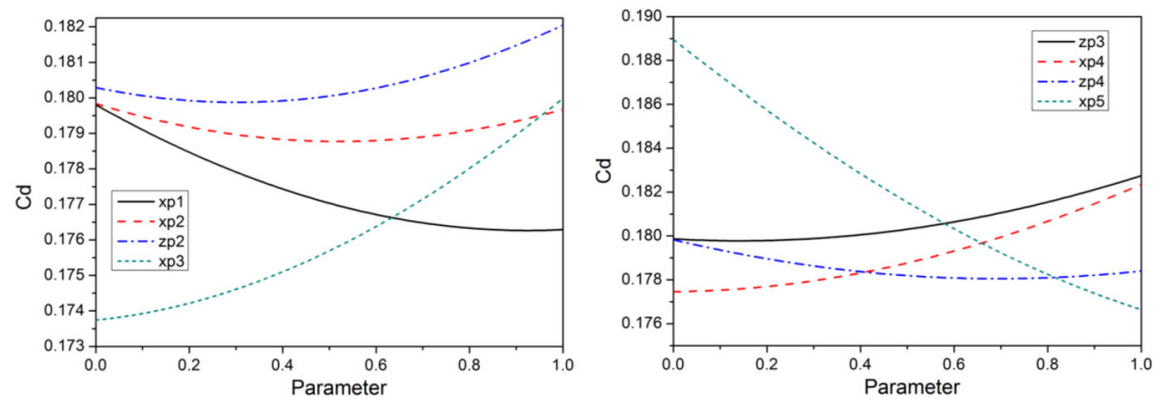

Fig. 22 Relationships between aerodynamic drag and the design variables that control the shape of the cowcatcher

cowcatcher could better attach on the surface and flow separation could be weakened.

As shown in Fig. 22, nonlinear relationships could be observed between aerodynamic drag and the design variables that control the shape of the cowcatcher, of which $x_{p 3}$ and $x_{p 5}$ get stronger influence. It can be seen from Fig. 4 that $x_{p 3}$ controls the concavity of the cowcatcher and $x_{p 5}$ controls the position of the bottom end of the cowcatcher. When $x_{p 3}$ and $x_{p 5}$ vary, strong influence could be detected on the high pressure zone in front of the cowcatcher. $x_{p 3}$ is linearly increasing with 

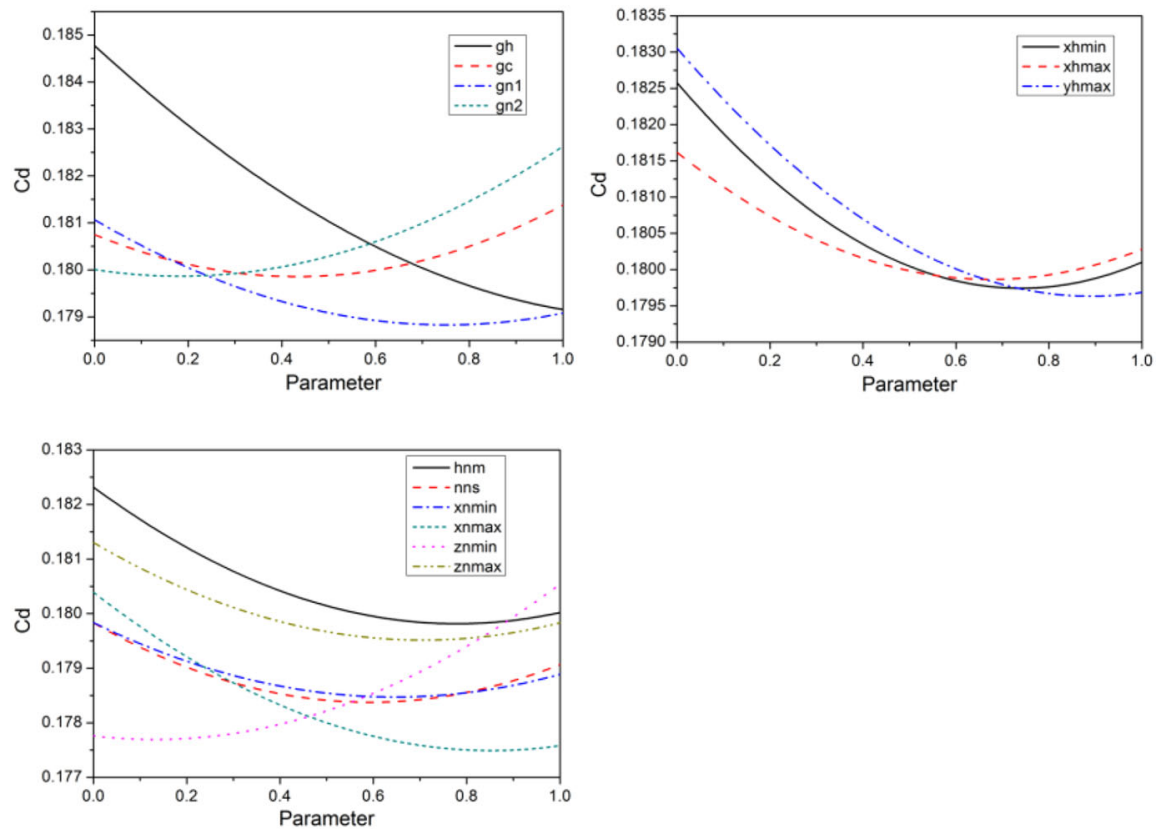

Fig. 23 Relationships between aerodynamic drag and the design variables that control the cab window and the drainage

aerodynamic drag. As $x_{p 3}$ increases, the concavity of the cowcatcher strengthens, resulting in higher pressure at the stagnation point. As a result, for the design of cowcatchers, the concavity of the cowcatcher should be weakened as much as possible as long as the basic function of the cowcatcher is ensured. $x_{p 5}$ linearly decreases with aerodynamic drag. As $x_{p 5}$ increases, the bottom of the cowcatcher tends to be backward, so that the intensity of the high pressure zone tends to be weakened. Considering the influences by $x_{p 3}$ and $x_{p 5}$, a smooth cowcatcher with no disturbance to the flow could be greatly helpful to reduce the drag.

As shown in Fig. 23, little influence could be observed between aerodynamic drag and the design variables that control the cab window and the drainage. However, nonlinear relationship could still be found. $g h$ is monotonically decreasing with the drag. Since $g h$ controls the view sight of the driver, the view of the driver should be firstly ensured and then the height of the cab window could be lowered. $g c$ controls the shape of the borders of the cab window. As $g c$ is big enough, the shape of the cab window tends to be square. As $g c$ is small enough, the shape of the cab window tends to be fusiform. A value between two extremes for $g c$ could result in smaller aerodynamic drag. $g n_{1}$ and $g n_{2}$ control the transition between the cab window and the nose in the width direction and length direction respectively. Big values of $g n_{1}$ and $g n_{2}$ will result in smooth transitions between the cab window and the nose. However, the surface area of the cab window will be reduced. It can be derived from Fig. 23 that smooth transition between the cab 
window and the nose could result in smaller drag. The position of the cab window is mainly controlled by $x_{\text {hmin }}, x_{\text {hmax }}$ and $y_{\text {hmax }}$. Tiny adjustment of the three variables could reduce the drag, but no big effect exists. Therefore, the location of the cab window should mainly consider the view of the driver and the placement of inner equipment. Among the 6 variables that control the drainage, $x_{n \max }$ and $z_{\text {nmin }}$ are the most important variables, which control the length and width of the drainage, respectively. As $x_{\text {nmax }}$ increases, the drainage extends backward, so that the drag decreases. As the increase of $z_{\text {nmax }}$ and decrease of $z_{\text {nmin }}$, the entrance and exit of the drainage enlarges (bigger size could be observed at the exit), also leading to a smaller drag. In order to reduce the drag, the drainage should be designed gradually widened from the entrance to the exit. Meanwhile, smooth transition between the drainage and the nose should be ensured.

\section{Conclusions}

In order to promote the application of parametric design approaches for the design of the nose shape of high speed trains, and improve the design efficiency of nose shape, a parametric method for nose shape has been proposed in the present paper by combining the VMF parametric method, NURBS curve method and discrete control point method. Based on this approach, a multi-objective aerodynamic optimization has been performed. The volume of the nose and aerodynamic drag of the whole train have been chosen as the constraints. Several conclusions have been obtained.

(1) The parametric design approach proposed in the present paper could describe the three-dimensional shape of the nose in detail. Totally different shape could be obtained by adjusting the design variables. It will aid in the concept design of nose shape and improving the optimization efficiency.

(2) For high dimensional optimization problems, the SVM regression model based on the multi-points criterion could accurately describe the nonlinear relationship between the design variables and objectives, and could be generally utilized in other fields. The PSO algorithm adopted in the present paper gets excellent capability in global optimization, especially in high dimensional multiobjective problems.

(3) Aerodynamic performance has been greatly improved for both the simplified model and the real model. For the real train model, the total drag of the optimal train has been reduced by $13.62 \%$, in which the drag of the leading car has been reduced by $25.41 \%$ and the drag of the trailing car has been reduced by $8.99 \%$. The lift of the trailing car has been reduced by $9.84 \%$.

(4) With the help of SVR model, nonlinear relationships could be obtained between aerodynamic drag and the design variables, which could shed light on the engineering design and optimization.

Acknowledgments This work was supported by 973 program under 2011CB711100 and National Natural Science Foundation of China under 11302233. And Computing Facility for Computational Mechanics Institute of Mechanics, Chinese Academy of Sciences is gratefully acknowledged. 


\section{References}

Cui K, Wang XP, Hu SC et al (2012) Shape optimization of high-speed train with the speed of $500 \mathrm{kph}$. Lecture notes in electrical engineering. In: Proceedings of the 1 st international workshop on highspeed and intercity railways, vol. 148. Springer, Heidelberg, pp 187-197

Hu X, Eberhart R (2002) Multi-objective optimization using dynamic neighborhood particle swarm optimization. In: Proceedings of the IEEE CEC. pp 1667-1681

Jongsoo Lee JK (2008) Approximate optimization of high-speed train nose shape for reducing micropressure wave. Ind Appl 35:79-87

Kennedy J, Eberhart RC (1995) Partical swarm optimization. In: Proceeding of the 1995 IEEE international conference on neural network, Perth, pp 1942-1948

Krajnovic S (2009) Shape optimization of high-speed trains for improved aerodynamic performance. Proc Inst Mech Eng Part F 223(5):439-452

Krajnovic S, Helgason E, Hafsteinsson H (2012) Aerodynamic shape optimization of high-speed trains. In: Proceedings of the first international conference on railway technology: research, development and maintenance, civil-comp press, Stirlingshire

Ku Y-C, Rho J-H, Yun S-H et al (2010a) Optimal cross-sectional area distribution of a high-speed train nose to minimize the tunnel micro-pressure wave. Struct Multidisc Optim 42:965-976

Ku Y-C, Kwak M-H, Park H-I, Lee D-H (2010b) Multi-objective optimization of high-speed train nose shape using the vehicle modeling function. In: 48th AIAA aerospace sciences meeting, Orlando

Li X (2003) A non-dominated sorting particle swarm optimizer for multiobjective optimization. In: Proceeding of genetic and evolutionary computation GECCO 2003: genetic and evolutionary computation conference, Springer, Berlin, pp 37-48

Moore J, Chapman R (1999) Application of particle swarm to multi-objective optimization. Department of Computer Science and Software Engineering, Auburn University, Auburn

Parsopoulos KE, Vrahatis MN (2002) Particle swarm optimization method in multiobjective problems. In: Proceedings of the ACM symposium on applied computing. Madrid, pp 603-607

Parsopoulos KE, Tasoulis DK, Vrahatis MN (2004) Multiobjective optimization using parallel vector evaluated particle swarm optimization. In: Proceedings of the IASTED international conference on artificial intelligence and applications (AIA 2004), vol 2. ACTA Press, Innsbruck, pp 823-828

Peng X (2010) TSVR: an efficient twin support vector machine for regression. Neural Netw 23(3):365-372

Rho JH, Ku YC, Yun SH et al (2009) Development of vehicle modeling function for 3-dimensional shape optimization. J Mech Des 131(12):121004-1-10

Shao YH, Zhang CH, Yang ZM et al (2013) An $\varepsilon$-twin support vector machine for regression. Neural Comput Appl 23(1):175-185

Yao S, Guo D, Yang G (2012a) Three-dimensional optimization design of high-speed train nose based on GA-GRNN. Sci China Technol Sci 55(11):3118-3130

Yao S, Guo D, Sun Z et al (2012b) Multi-objective optimization of the streamlined head of high-speed trains based on the Kriging model. Sci China Technol Sci 55(12):3494-3508

Yao S, Guo D, Sun Z et al (2014) Optimization design for aerodynamic elements of high speed trains. Comput Fluids 95:56-73

Vapnik VN (1998) Statistical learning theory. Wiley, New York

Vytla VV, Huang PG, Penmetsa RC (2010) Multi objective aerodynamic shape optimization of high speed train nose using adaptive surrogate model. In: AIAA-2010-4383 